\title{
Regulation of wingless-type MMTV integration site family (WNT) signalling in pancreatic islets from wild-type and obese mice
}

\author{
J. Krützfeldt $•$ M. Stoffel
}

Received: 7 July 2009 /Accepted: 15 September 2009/Published online: 8 November 2009

(C) Springer-Verlag 2009

\begin{abstract}
Aims/hypothesis TCF7L2 is a type 2 diabetes susceptibility gene and downstream effector of canonical wingless-type MMTV integration site family (WNT) signalling. However, it is unknown whether this pathway is active in adult pancreatic islets in vivo, and whether it is regulated in obesity.

Methods We analysed activation of endogenous WNT signalling in the endocrine pancreas from wild-type and obese mice (ob/ob) using a reporter transgene (Topgal). Regulation of WNT signalling was compared using gene chip experiments from isolated pancreatic islets. Activation of canonical WNT signalling in pancreatic islets and the mouse beta cell line MIN6 was measured using immunoblotting for cytosolic $\beta$-catenin.

Results Endogenous canonical WNT signalling was absent in the adult endocrine pancreas in both wild-type and obese mice. We identified WNT4 as an abundant WNT signalling molecule in adult pancreatic islets that is induced in two different insulin-resistant mouse models. Increased expression of WNT4 inhibited canonical WNT signalling in pancreatic islets and MIN6 cells.
\end{abstract}

Electronic supplementary material The online version of this article (doi:10.1007/s00125-009-1578-2) contains supplementary material, which is available to authorised users.

J. Krützfeldt $\cdot$ M. Stoffel

Laboratory of Metabolic Diseases, The Rockefeller University,

New York, NY, USA

Present Address:

J. Krützfeldt $\cdot$ M. Stoffel $(\bowtie)$

ETH Zurich, Institute for Molecular Systems Biology,

Laboratory of Metabolic Diseases,

HPT E73, Wolfgang-Pauli Str. 16,

CH-8093 Zurich, Switzerland

e-mail: stoffel@imsb.biol.ethz.ch
Conclusions/interpretation Canonical WNT signalling is not active in adult beta cells in vivo. WNT4 provides a potential mechanism for suppression of canonical WNT signalling in obese mice.

Keywords $\beta$-Catenin $\cdot$ Microarray $\cdot o b / o b \cdot$ Pancreatic islets · Topgal · Type 2 diabetes $\cdot$ WNT4
Abbreviations
GFP Green fluorescent protein
GSK3 $\beta$ Glycogen synthase kinase-3
LEF Lymphoid enhancer factor
PDX1 Pancreatic and duodenal homeobox 1
TCF T cell factor
WNT Wingless-type MMTV integration site

\section{Introduction}

Among common type 2 diabetes risk genes, polymorphisms in the TCF $7 L 2$ gene provide the strongest disease association [1]. The gene encodes for transcription factor 7-like-2, which interacts with $\beta$-catenin as a downstream effector of the WNT signalling pathway. Canonical WNT signalling involves binding of WNT molecules to cell-surface receptors called frizzled and to co-receptors, the lipoprotein receptor-related protein. Binding of WNT molecules leads to inhibition of glycogen synthase kinase-3 (GSK3 $\beta$ ), which results in the stabilisation of cytosolic $\beta$-catenin. Subsequently, $\beta$-catenin translocates to the nucleus where it activates WNT target genes via binding to $\mathrm{T}$ cell factor (TCF)/lymphoid enhancer factor (LEF) promoter elements.

Polymorphisms in TCF7L2 are associated with impaired insulin secretion $[2,3]$ suggesting an important role of the 
WNT/ $\beta$-catenin pathway in pancreatic islets. However, lossof-function experiments in animal models have revealed conflicting results. Transgenic expression of an inhibitor of WNT signalling (Pdx1-tTa/TRE-axin) revealed a decrease of pancreatic beta cell mass by $50-60 \%$ and impaired glucose tolerance [4], but attenuation of WNT signalling in a different transgenic mouse model (Pdx1/Frz8CD) showed normal glucose tolerance and a relative increase in endocrine cell numbers compared with exocrine tissue [5]. Furthermore, beta cell-specific deletion of $\beta$-catenin (Pdx 1-Cre,Catnb ${ }^{\text {lox/lox }}$ ) did not alter islet architecture or glucose tolerance at 6 or 12 weeks of age $[6,7]$, but deletion of $\beta$-catenin in pancreatic islets at later stages of development (RIP-Cre,Catnb ${ }^{\text {lox/lox }}$ ) induced about $70 \%$ perinatal lethality, possibly because of hypoglycaemia [8].

Clearly, more research is needed to clarify whether WNT signalling is active in beta cells in vivo [9]. Therefore, we studied activation of canonical WNT signalling in pancreatic islets from adult wild-type and obese mice using a reporter transgene (Topgal) and analysed regulation of the $\mathrm{WNT} / \beta$-catenin signalling pathway at the molecular level using gene chip experiments.

\section{Methods}

Mice All mice were housed as described previously [10]. Principles of laboratory animal care (NIH, revised 1985) were followed. $\mathrm{ob} / \mathrm{ob}$ mice $\left(\mathrm{Lep}^{-/}\right)$were purchased from Jackson Laboratories (Bar Harbor, ME, USA). Topgal mice were obtained from E. Fuchs (Rockefeller University, New York, USA). For gene chip studies, age of mice was $9.7 \pm 0.2(n=36)$ vs $8.6 \pm 0.1$ weeks $(n=117, p<0.05)$; random glucose was $12.6 \pm 0.7(n=36)$ vs $10.0 \pm 0.2 \mathrm{mmol} / \mathrm{l}(n=107, p<0.05)$; and random insulin was 4,669.1 $\pm 1,567.8(n=10)$ vs $68.8 \pm$ $10.3 \mathrm{pmol} / \mathrm{l}(n=10, p<0.05)$; all $o b / o b$ vs control, respectively.

Cell culture Mouse beta cell line MIN6 cells were grown as described earlier [10]. Adenoviruses were generated using the Rapid Adenovirus Production System (Viraquest, North Liberty, USA), employing the pVQ-CMV-K-Npa shuttle vector containing PCR-cloned murine Wnt4 cDNA.

Isolation of pancreatic islets and RT-PCR analysis Pancreatic islets were isolated by collagenase digestion and differential centrifugation as previously described [10]. Total RNA was extracted from double hand-picked islets using Trizol reagent (Invitrogen, Carlsbad, CA, USA) and RT-PCR carried out for semiquantitative gene expression analysis as described earlier [10].

Oligonucleotide microarray analysis Microarray analysis was performed as previously described using $10 \mu \mathrm{g}$ total
RNA from isolated islets per chip experiment [11]. Duplicate arrays were performed for the $o b / o b$ group and controls, respectively, and genes were considered differentially regulated if a significant $p$ value was obtained according to the Affymetrix software (Santa Clara, CA, USA) in all of the four possible pair-wise comparisons.

$\beta$-Galactosidase staining Pancreases were perfused via the bile duct with fixing solution $\left(100 \mathrm{mmol} / 1 \mathrm{NaPO}_{4}, \mathrm{pH} 7.3\right.$, $0.2 \%$ [vol./vol.] glutaraldehyde, $5 \mathrm{mmol} / 1$ EGTA, $2 \mathrm{mmol} /$ $1 \mathrm{MgCl}_{2}$ ) and immediately placed in ice-cold PBS containing $10 \%$ (wt/vol.) sucrose. Sections of $4 \mu \mathrm{m}$ were mounted on slides and fixed for $2 \mathrm{~min}$ at room temperature in fixing solution. After washing, sections were stained overnight in the dark at $37^{\circ} \mathrm{C}$ in staining solution $(1 \mathrm{mg} / \mathrm{ml} \mathrm{X}$-gal [5-bromo-4chloro-3-indolyl- $\beta$-D-galactoside], $2.1 \mathrm{mg} / \mathrm{ml} \mathrm{K}_{4} \mathrm{Fe}$ $(\mathrm{CN})_{6} \cdot 3 \mathrm{H}_{2} \mathrm{O}, 1.64 \mathrm{mg} / \mathrm{ml} \mathrm{K}{ }_{3} \mathrm{Fe}(\mathrm{CN})_{6}, 2 \mathrm{mmol} / 1 \mathrm{MgCl}_{2}$, $0.04 \%$ [wt/vol.] deoxycholate, $0.08 \%$ [vol./vol.] NP-40, $100 \mathrm{mmol} / 1 \mathrm{NaPO}_{4}, \mathrm{pH} 7.3$ ).

Western blotting For detection of cytosolic $\beta$-catenin, MIN6 cells or pancreatic islets were harvested in TRISbuffered saline (pH 7.5) containing $2 \mathrm{mmol} / \mathrm{l}$ dithiothreitol, $2 \mathrm{mmol} / \mathrm{l}$ phenylmethylsulfonyl fluoride, $5 \mathrm{mmol} / \mathrm{l}$ EDTA and $10 \%$ (vol./vol.) protease cocktail (Roche, Indianapolis, USA). Cells were lysed by one freeze/thaw cycle and subjected to ultracentrifugation $\left(100,000 \mathrm{~g}, 90 \mathrm{~min}, 4^{\circ} \mathrm{C}\right)$. The resulting cytosolic fractions were separated by $10 \%$ (wt/vol.) SDS-PAGE and transferred onto nylon membranes. $\beta$-Catenin and GSK3 $\beta$ were visualised using monoclonal antibodies (anti- $\beta$-catenin [Sigma Aldrich, St Louis, USA], anti-GSK $3 \beta$ [BD Transduction Laboratories, Lexington, Kentucky, USA]), peroxidase-labelled second antibodies and enhanced chemiluminescence (PerkinElmer, Waltham, MA, USA). Anti-WNT4 polyclonal antibodies and recombinant WNT3A were purchased from R\&D Systems (Minneapolis, USA).

\section{Results}

Absence of activated $W N T / \beta$-catenin signalling in pancreatic islets in wild-type and obese mice in vivo To detect canonical wnt signalling, we used the transgenic reporter mouse model Topgal, which harbours the LacZ gene under a TCF/ LEF1-responsive promoter element. Endogenous activation of canonical wnt signalling in these mice results in $\beta$ galactosidase activity in all WNT-responsive cells. Immunohistochemistry demonstrated that marked $\beta$-galactosidase activity was detected in close proximity to islets within small vessels of the pancreas (Fig. 1a). We crossed the Topgal transgene into mice that express green fluorescent 
a
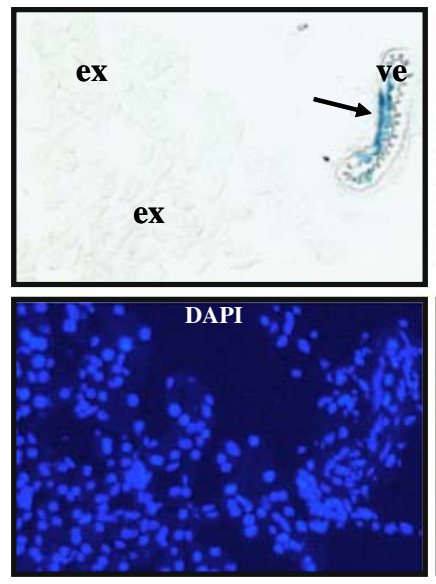

b
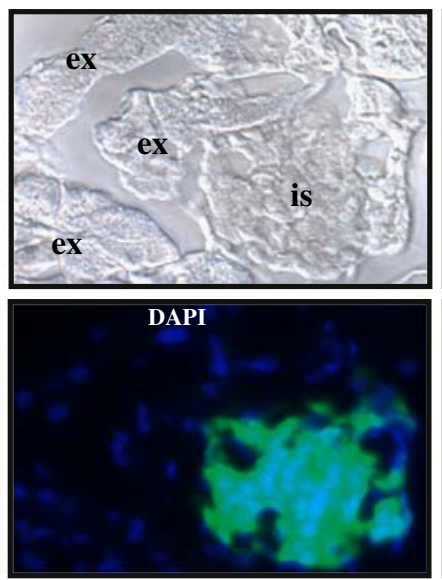

C
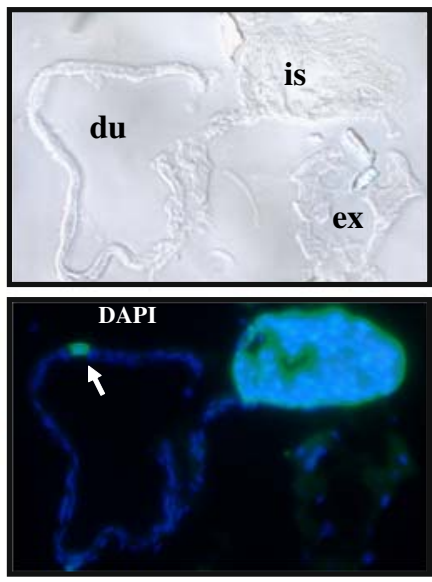

d
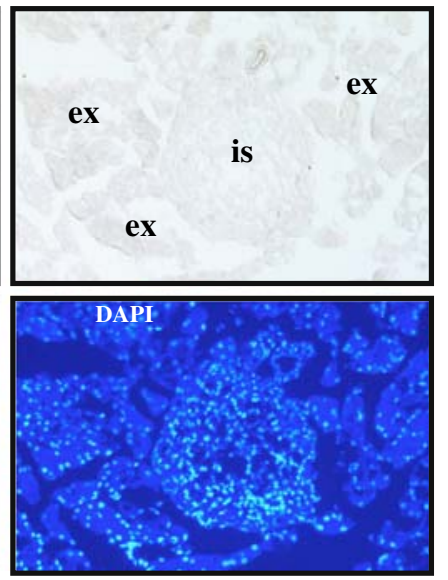

Fig. 1 Activation of the WNT/ $\beta$-catenin pathway in small pancreatic vessels, but not in the endocrine pancreas in vivo. Activation of the $\mathrm{WNT} / \beta$-catenin pathway was visualised in cryosections from the pancreas of 2-month-old Topgal/wild-type (a), Topgal/PDX-GFP (b, c) or Topgal/ob/ob (d) mice by $\beta$-galactosidase staining (upper

protein (GFP) under the control of the pancreatic and duodenal homeobox 1 (PDX1) promoter (PDX-GFP) to label pancreatic beta cells and PDX1-positive duct cells. However, PDX1-positive cells in pancreatic islets and

panels). Lower panel shows fluorescence from the same sections with nuclear DAPI staining. Black arrow, $\beta$-galactosidase activity in a small blood vessel. White arrow, PDX-positive duct cell with absent $\beta$-galactosidase activity. du, pancreatic duct; ex, exocrine pancreas; is, islet; ve, blood vessel

pancreatic ducts lack $\beta$-galactosidase activity (Fig. 1b, c). Lack of $\beta$-galactosidase activity in pancreatic islets was not caused by technical constraints, as $\beta$-galactosidase was readily detectable in pancreatic islets from mutant Foxa 2

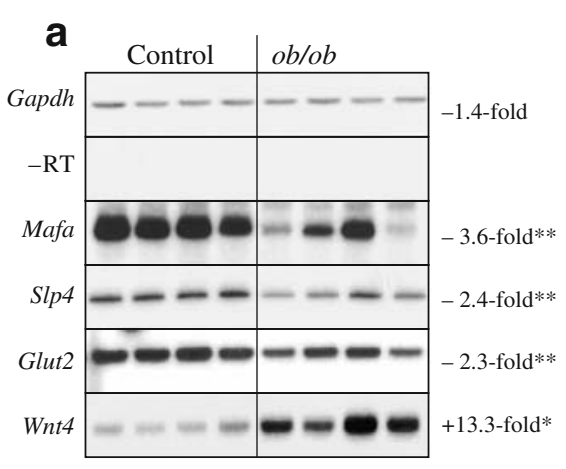

C

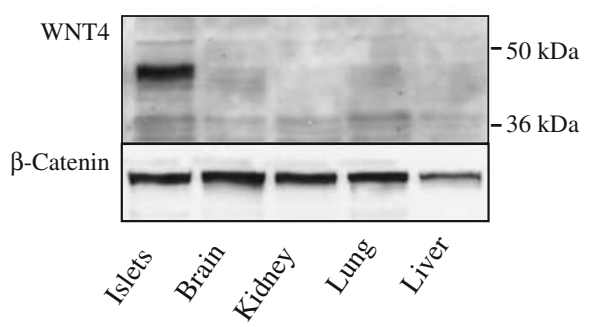

Fig. 2 Increased expression of Wnt4 in pancreatic islets from insulinresistant mice. cDNA from isolated pancreatic islets from control mice or two different insulin-resistant mouse models (ob/ob, aP2-nSREBP1c) was analysed using semiquantitative RT-PCR (a, b). Gapdh was amplified to demonstrate equal amounts of cDNA. Quantitative measurements were obtained by densitometry and expressed as fold change vs control. ${ }^{*} p<0.05, * * p<0.01$ analysed by two-tailed
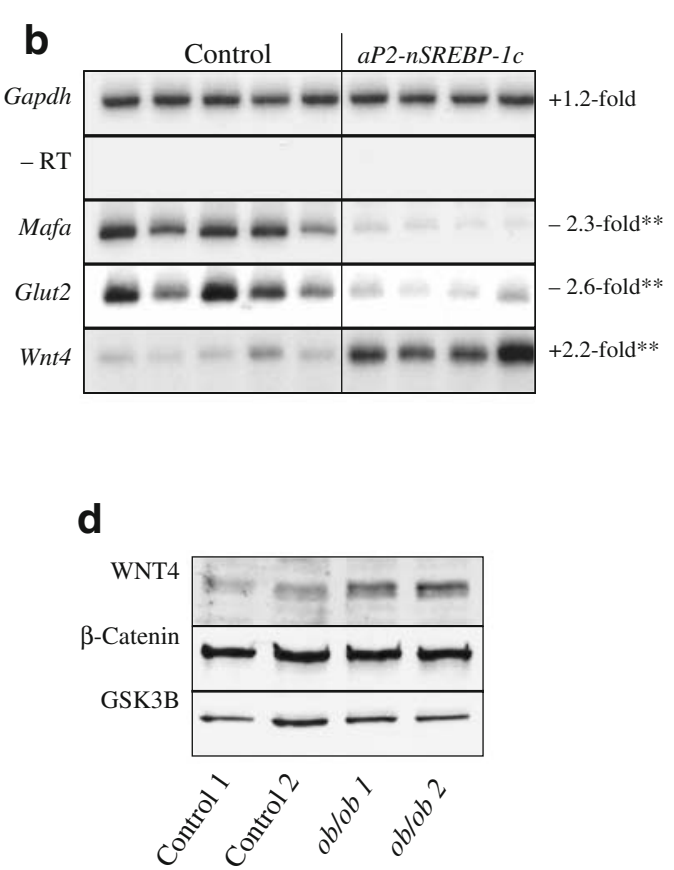

student's $t$ test. $-\mathrm{RT}$, without reverse transcriptase. c, d Amount of WNT4 protein was measured by immunoblotting in whole cell lysates (50 $\mathrm{\mu g}$ protein or 150 islets, respectively), antibodies against $\beta$ catenin and GSK $3 \beta$ were used as loading controls. Expression of WNT4 was compared between isolated pancreatic islets and different adult mouse tissues in wild-type mice (c) and between pancreatic islets isolated from control or $o b / o b$ mice (d) 
mice [11] that have one inactivated Foxa 2 allele by targeted insertion of the $L a c Z$ gene (data not shown). Next, we crossed the Topgal transgene into obese mice $(o b / o b)$. Similarly to our observations in wild-type mice, activation of the $\mathrm{WNT} / \beta$-catenin pathway was not detected in pancreatic islets from obese mice (Fig. 1d).

WNT4 is an abundant protein in pancreatic islets and upregulated in insulin-resistant mouse models To investigate the molecular basis for the absence of activated WNT signalling in pancreatic islets in vivo we performed gene chip experiments. We collected pancreatic islets from $o b / o b$ mice and control mice (animal characteristics in the Methods). We found that 169 genes were consistently regulated, 105 genes were increased and 64 genes were decreased (Electronic supplementary material [ESM] Table 1). Importantly, we confirmed downregulation of several genes in obese mice that are known to be decreased in beta cells during hyperglycaemia, i.e. Glut2 (also known as Slc2a2), Mafa, Slp4 (also known as Sytl4) and G3pdh. Two members of the WNT signalling pathway, WNT4 and frizzled-related protein, were upregulated in pancreatic islets from obese mice. We confirmed regulation of Wnt 4 by semiquantitative RTPCR (Fig. 2b). In addition, Wnt4 expression was also increased in islets from a different insulin-resistant mouse model, lipodystrophic mice that lack adipose tissue $(a P 2$ $n S R E B P-1 c$ [11]) (Fig. 2c). Western blot analysis of different adult mouse tissues detected endogenous WNT4 protein only in pancreatic islets (Fig. 2b). Consistent with the regulation at the mRNA level, WNT4 protein is more abundant in islets from obese mice compared with lean controls (Fig. 2d). Together, these data demonstrate that WNT4 protein is enriched in pancreatic islets and its expression is increased in two different insulin-resistant mouse models.

WNT4 is an inhibitor of the canonical WNT signalling pathway in pancreatic islets WNT members are not intrinsically canonical or non-canonical, but their signalling is dictated by the distinct set of receptors within a certain cell type [12]. To address the impact of WNT4 on the $\mathrm{WNT} / \beta$-catenin pathway we overexpressed WNT4 in isolated pancreatic islets using an adenovirus system. No stabilisation of $\beta$-catenin was noted when WNT4 levels were increased in pancreatic islets (Fig. 3a). To characterise the $\mathrm{WNT} / \beta$-catenin pathway in beta cells in response to WNT4 in more detail we analysed MIN6 cells. Consistent with the results obtained in pancreatic islets, adenoviral WNT4 did not activate the $\mathrm{WNT} / \beta$-catenin pathway in MIN6 cells (Fig. 3b). In contrast, WNT4 efficiently inhibited the stabilisation of cytosolic $\beta$-catenin that had been induced by recombinant WNT3A. This inhibition was specific for the WNT receptor, since receptor-independent stabilisation of cytosolic $\beta$-catenin by the GSK3 $\beta$ inhibitor lithium chloride was not altered by Wnt4 (Fig. 3c). These results demonstrate that WNT4 is an inhibitor of the WNT/ $\beta$-catenin pathway in pancreatic islets and beta cells.

\section{Discussion}

The major finding of our study is the absence of activated canonical WNT signalling in adult pancreatic islets in vivo even in the presence of obesity and hyperinsulinaemia. This was surprising, since canonical wnt signalling can be activated by adipocyte-derived WNT signalling molecules [13] and high insulin levels [14]. Importantly, our study identifies a potential mechanism for this discrepancy. In adult mice, WNT4 is an abundant WNT signalling molecule in pancreatic islets, and it is not detected in several other adult mouse tissues. In the context of the pancreatic islet, WNT4 is a specific inhibitor of canonical wnt signalling and robust upregulation of WNT4 was observed in pancreatic islets from two different insulinresistant mouse models. Thus, WNT4 can provide a molecular link to prevent cross-talks between pancreatic islets and adipocyte-derived WNT signalling molecules (e.g. from adipocytes within the pancreas [13]) and between pancreatic islets and hyperinsulinaemia. Higher WNT4 expression in insulin-resistant mice might also induce non-canonical wnt signalling pathways, which, however, are diverse and poorly defined in mammalian tissues. a

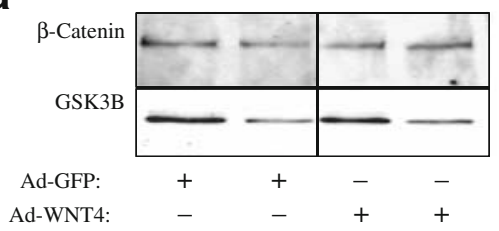

b

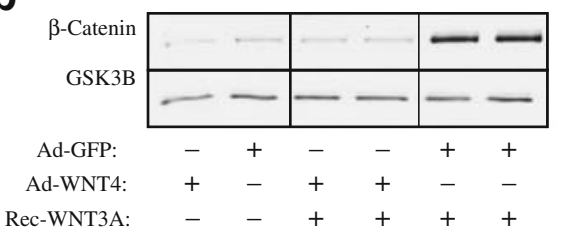

C

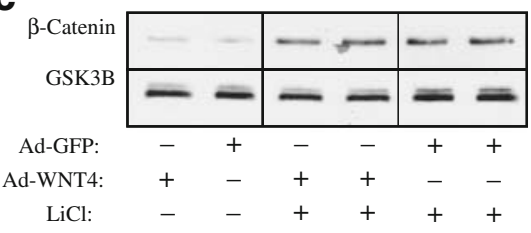

Fig. 3 WNT4 inhibits canonical WNT signalling in pancreatic islets at the receptor level. Activation of the $\mathrm{WNT} / \beta$-catenin pathway was measured by immunoblotting of $\beta$-catenin in cytosolic fractions. a Comparison of cytosolic $\beta$-catenin in isolated pancreatic islets after infection with control virus (Ad-GFP) or virus that overexpresses WNT4 (Ad-WNT4). b, $\mathbf{c}$ Effect of adenoviral WNT4 on activation of the WNT/ $\beta$-catenin pathway in MIN6 cells by recombinant WNT3A (Rec-WNT3A, $30 \mathrm{ng} / \mathrm{ml})(\mathbf{b})$ or lithium chloride $(\mathrm{LiCl}, 10 \mathrm{mmol} / \mathrm{l})(\mathbf{c})$ 
Previous studies reported different results on the impact of wnt/ $\beta$-catenin signalling in beta cells. Deletion of $\beta$ catenin early in development (Pdx1-Cre,Catnb ${ }^{\text {lox/lox }}$ ) did not show alterations in islet development and normal glucose tolerance later during adult life $[6,7]$. In contrast, deletion of $\beta$-catenin in pancreatic islets at later stages of development (RIP-Cre,Catnb ${ }^{l o x / l o x}$ ) induced about $70 \%$ perinatal lethality and the surviving mice showed only mild glucose intolerance later in adulthood [8]. Beta cells might be specifically susceptible to loss of $\beta$-catenin around the time of birth, which is consistent with activation of endogenous canonical wnt signalling in the endocrine pancreas of newborn mice [6]. However, $\beta$-catenin signalling in beta cells might be dispensable later in life since endogenous wnt signalling is not activated and possibly even actively suppressed. Genes that are downstream effectors of canonical WNT signalling such as TCF7L2 could impact pancreatic islets through $\beta$-catenin-independent aspects or at earlier stages of development.

Acknowledgements We thank L. Castelo-Soccio and A.M.C. Brown (Weill Cornell Medical College, New York, USA) for technical advice. The work was supported by a grant to J. Krützfeldt from Deutsche Forschungsgemeinschaft (KR 2224/1-1).

Duality of interest The authors declare that there is no duality of interest associated with this manuscript.

\section{References}

1. Pearson ER (2009) Translating TCF7L2: from gene to function. Diabetologia 52:1227-1230
2. Lyssenko V, Lupi R, Marchetti P et al (2007) Mechanisms by which common variants in the TCF7L2 gene increase risk of type 2 diabetes. J Clin Invest 117:2155-2163

3. Schäfer SA, Tschritter O, Machicao F et al (2007) Impaired glucagon-like peptide-1-induced insulin secretion in carriers of transcription factor 7-like 2 (TCF7L2) gene polymorphisms. Diabetologia 50:2443-2450

4. Rulifson IC, Karnik SK, Heiser PW et al (2007) Wnt signalling regulates pancreatic beta cell proliferation. Proc Natl Acad Sci U S A 104:6247-6252

5. Papadopoulou S, Edlund H (2005) Attenuated Wnt signalling perturbs pancreatic growth but not pancreatic function. Diabetes $54: 2844-2851$

6. Dessimoz J, Bonnard C, Huelsken J, Grapin-Botton A (2005) Pancreas-specific deletion of beta-catenin reveals Wnt-dependent and Wnt-independent functions during development. Curr Biol 15:1677-1683

7. Murtaugh LC, Law AC, Dor Y, Melton DA (2005) Beta-catenin is essential for pancreatic acinar but not islet development. Development 132:4663-4674

8. Dabernat S, Secrest P, Peuchant E, Moreau-Gaudry F, Dubus P, Sarvetnick N (2009) Lack of beta-catenin in early life induces abnormal glucose homeostasis in mice. Diabetologia 52:1608-1617

9. Welters HJ, Kulkarni RN (2008) Wnt signalling: relevance to betacell biology and diabetes. Trends Endocrinol Metab 19:349-355

10. Akpinar P, Kuwajima S, Krützfeldt J, Stoffel M (2005) Tmem27: a cleaved and shed plasma membrane protein that stimulates pancreatic beta cell proliferation. Cell Metab 2:385-397

11. Wolfrum C, Asilmaz E, Luca E, Friedman JM, Stoffel M (2004) Foxa2 regulates lipid metabolism and ketogenesis in the liver during fasting and in diabetes. Nature 432:1027-1032

12. van Amerongen R, Mikels A, Nusse R (2008) Alternative wnt signalling is initiated by distinct receptors. Sci Signal 1:re9

13. Schinner S, Ulgen F, Papewalis C et al (2008) Regulation of insulin secretion, glucokinase gene transcription and beta cell proliferation by adipocyte-derived Wnt signalling molecules. Diabetologia 51:147-154

14. Yi F, Sun J, Lim GE, Fantus IG, Brubaker PL, Jin T (2008) Cross talk between the insulin and Wnt signalling pathways: evidence from intestinal endocrine L cells. Endocrinology 149:2341-2351 